Ewa Laskowska1®, Piotr Michalski2®), Łukasz Pietrzykowski², Michał Kasprzak ${ }^{1}$, Agata Kosobucka², Klaudyna Grzelakowska ${ }^{3}$, Krzysztof Buczkowski ${ }^{4}$, Piotr Jankowski ${ }^{5}$, Zbigniew Gąsior ${ }^{6}$, Dariusz Kosior ${ }^{7}$, Karol Kamiński ${ }^{8}$, Aldona Kubica ${ }^{2}$

${ }^{1}$ Department of Cardiology and Internal Medicine, Collegium Medicum, Nicolaus Copernicus University, Bydgoszcz, Poland

2Department of Health Promotion, Collegium Medicum, Nicolaus Copernicus University, Bydgoszcz, Poland

${ }^{3}$ Faculty of Medicine, Collegium Medicum in Bydgoszcz, Nicolaus Copernicus University, Bydgoszcz, Poland

${ }^{4}$ Department of Family Medicine, Nicolaus Copernicus University, Torun, Poland

${ }^{5}$ Department of Epidemiology and Health Promotion, School of Public Health, Centre of Postgraduate Medical Education, Warsaw, Poland

${ }^{6}$ Department of Cardiology, Medical University of Silesia, Katowice, Poland

${ }^{7}$ Department of Cardiology and Hypertension with Electrophysiological Lab, Central Research Hospital the Ministry of the Interior

and Administration, Warsaw, Poland

${ }^{8}$ Department of Population Medicine and Civilization Diseases Prevention, Medical University of Bialystok, Poland

\title{
Implementation of therapeutic recommendations in high cardiovascular-risk patients. The Polish population of EUROASPIRE V survey
}

\section{Corresponding author:}

Ewa Laskowska, Department of Cardiology and Internal Medicine, Collegium Medicum, Nicolaus Copernicus University, Bydgoszcz, Poland,

e-mail: ewa.laskowska@cm.umk.pl
Medical Research Journal 2021: Volume 6, Number 3, 230-236 10.5603/MRJ.a2021.0045

Copyright (C) 2021 Via Medica ISSN 2451-2591

e-ISSN 2451-4101

\begin{abstract}
:
Introduction: Poor medication adherence is associated with unsatisfactory health outcomes, elevated mortality, and high costs of medical care. This study aimed to assess the implementation of therapeutic recommendations in high cardiovascular-risk patients based on self-report questionnaires.

Material and methods: The study included 194 patients from the Cardiology Outpatient Clinic. Two self-reported questionnaires were used to assess medication adherence: the Medication Adherence Questionnaire (MAQ) and the Adherence in Chronic Diseases Scale (ACDS). Results: Antihypertensive drugs were prescribed to $65.46 \%$ of the patients. According to the MAQ, $54.33 \%$ of them reported high adherence, $21.26 \%$ medium adherence, and $24.41 \%$ low adherence to the treatment. Lipid-lowering drugs were prescribed to $46.39 \%$ of the patients, all of whom were treated with statins. Among this group, 34.44\% reported high adherence, $27.78 \%$ medium adherence, and $37.78 \%$ low adherence to pharmacotherapy. According to the ACDS, the majority of patients (45.55\%) received a score indicating medium adherence (21-26 points), 39.27\% high adherence ( $>26$ points), and $16.75 \%$ low adherence to treatment ( $<21$ points). A high level of adherence was declared by $61.54 \%$ of the patients that reached the therapeutic goal of lipid-lowering therapy, defined as LDL-C of $<2.6 \mathrm{mmol} / \mathrm{L}$ $(<100 \mathrm{mg} / \mathrm{dL}$ ). On the other hand, among the patients whose LDL-C remained elevated, $23.44 \%$ declared high adherence to treatment. There were no significant differences in achieving the intended therapeutic goal of blood pressure (BP $\leq 140 / 90 \mathrm{mmHg}$ ) in the groups with high, medium and low adherence $(26.53 \%$ vs. $23.47 \%$ vs. $50.00 \% ; p=0.1880$ ).

Conclusion: Despite higher adherence to treatment in the patients with hypertension compared to patients with hyperlipidemia, the latter more often achieved the therapeutic goal. Declarations regarding high adherence to medication in the MAQ and in the ACDS are consistent in patients with hyperlipidemia and hypertension

Key words: adherence, self-report questionnaires, hyperlipidemia, hypertension
\end{abstract}

Med Res J 2021; 6 (3): 230-236 


\section{Introduction}

Poor medication adherence is associated with unsatisfactory health outcomes, elevated mortality, and high costs of medical care [1,2]. According to various studies, $30 \%$ to $50 \%$ of patients do not adhere to medical recommendations [3-8]. In secondary prevention of myocardial infarction, the adherence to pharmacotherapy decreases over time, for nearly all classes of drugs [3, 13]. The pharmacotherapy of hypertension, dyslipidemia, and diabetes reduces the risk of cardiovascular events [9]; yet, adherence to preventive pharmacotherapy is even lower compared to secondary prevention [9-12]. In a meta-analysis comparing adherence to pharmacotherapy of coronary heart disease in primary vs. secondary prevention, Naderi et al. [12] reported an adherence rate of $50 \%(\mathrm{Cl}, 45-56)$ and $66 \%(\mathrm{Cl}, 56-75)$, respectively $(p=0.012)$. Overall, the adherence in the study was $57 \%$ during a median treatment of 24 months [12].

A number of validated medication-adherence scales have been described in the literature [8, 13-15]. These scales, based on self-reported questionnaires, are low-cost and easy to use in routine clinical practice [8].

This study aimed to assess the implementation of therapeutic recommendations in a group of patients treated for hypertension, hyperlipidemia, and/or diabetes with the use of two self-report questionnaires.

\section{Material and methods}

The data were collected as a part of the EUROASPIRE V survey, a prospective observational study conducted between 2016 and 2017 in European countries. The study included 200 patients $(66.50 \%$ women and $33.50 \%$ men), with a mean age of 51.49 years, from the Cardiology Outpatient Clinic of the Department of Cardiology and Internal Medicine of the University Hospital No. 1 in Bydgoszcz, Poland. The following inclusion criteria were applied: adult (18-80 years old), diagnosed with hypertension, hyperlipidemia or diabetes, without documented cardiovascular diseases. All the patients provided written informed consent. Baseline characteristics of the patient population are presented in Tables 1 and 2.

The final analysis comprised 194 patients treated with one or more medications for a period of 6-24 months (lipid-lowering drugs, antihypertensive drugs, oral antidiabetic medicines, and/or insulin). Six of the patients were treated with a diet for diabetes, and therefore were excluded from the analysis.

\section{Questionnaires}

Two self-reported questionnaires were used to assess medication adherence: the Medication Adherence Questionnaire (MAQ) and the Adherence in Chronic Diseases Scale (ACDS) [13-15].

In the MAQ, the patients were asked to answer the question "How often do you take your medications as

Table 1. Characteristics of the study population

\begin{tabular}{lccccccc}
\hline Parametr & Mean & SD & Median & Q1 & Q3 & Min & Max \\
\hline Age [years] & 51.49 & 13.63 & 52.00 & 43.00 & 60.00 & 20.00 & 81.00 \\
SBP [mmHg] & 127.23 & 14.47 & 125.00 & 118.00 & 135.00 & 97.00 & 183.00 \\
DBP [mmHg] & 76.52 & 9.32 & 77.50 & 70.00 & 82.00 & 54.00 & 100.00 \\
Waist circumference [cm] & 88.01 & 12.20 & 87.00 & 80.00 & 95.50 & 54.00 & 126.00 \\
Height [cm] & 169.47 & 9.62 & 169.50 & 162.00 & 176.00 & 147.00 & 200.00 \\
Weight [kg] & 75.77 & 14.79 & 74.00 & 64.75 & 86.00 & 44.00 & 118.00 \\
BMI [kg/m2] & 26.35 & 4.06 & 26.00 & 23.90 & 28.73 & 17.10 & 42.20 \\
T-CH [mg/dL] & 217.42 & 41.78 & 214.85 & 190.84 & 241.69 & 105.60 & 344.50 \\
LDL-CH [mg/dL] & 129.09 & 38.13 & 127.10 & 103.80 & 154.55 & 42.20 & 253.00 \\
TG [mg/dL] & 121.04 & 71.32 & 106.80 & 79.96 & 137.50 & 40.20 & 630.90 \\
Glucose [mg/dL] & 100.60 & 19.74 & 97.60 & 90.75 & 106.35 & 52.20 & 207.70 \\
ACDS - score & 24.35 & 3.67 & 25.00 & 22.00 & 28.00 & 12.00 & 28.00 \\
\hline ACDS -Adner. & & & & &
\end{tabular}

ACDS — Adherence in Chronic Diseases Scale; BMI — body mass index; DBP — diastolic blood pressure; LDL-CH — low-density lipoprotein cholesterol; SBP — systolic blood pressure; SD — standard deviation; T-CH — total cholesterol; TG — triglyceride 
Table 2. Qualitative variables in the study population

\begin{tabular}{lcc}
\hline Parameter & Count & Percent \\
\hline Gender (female/male) & $133 / 67$ & $66.5 / 33.5$ \\
Hypertension & 127 & 63.5 \\
Hyperlipidemia & 90 & 45 \\
Diabetes & 41 & 20.5 \\
Smoking & 76 & 38 \\
Any pharmacotherapy & 194 & 97 \\
Antihypertensive drugs & 127 & 63.5 \\
Lipid-lowering drugs & 90 & 45 \\
Hypoglycemic drugs & 34 & 17 \\
\hline
\end{tabular}

prescribed by the doctor. Based on their response, they were then classified into one of three groups depending on the level of adherence: high (taking medication 100\% of the time as prescribed), medium ( $90 \%$ of the time), low $(75 \%$ of the time or less).

Another tool used to evaluate adherence to pharmacotherapy was the ACDS. This scale consists of 7 questions relating to behaviors influencing adherence, either directly (questions 1-5) or indirectly (questions 6 and 7). The maximum score in the ACDS is 32 points. A score above 26 points indicates high adherence, while a score under 21 points indicates low adherence; the remaining score range of $21-26$ points is classified as medium adherence [13-15].

\section{Statistics}

The statistical analysis was carried out using the Statistica 13.0 package (TIBCO Softwarelnc, California, USA). Continuous variables were presented as means with standard deviations, medians with interquartile range, minimum and maximum value. The Shapiro-Wilk test demonstrated the non-normal distribution of the investigated continuous variables.

Therefore, non-parametric tests were used for statistical analysis. For comparisons of ACDS scores and MAQ levels, the Kruskal-Wallis one-way analysis of variance and a multiple comparison test was used. Categorical variables were expressed as counts and percentages. Categorical variables were compared using the $\chi 2$ test. Results were considered significant at $p<0.05$.

\section{Results}

Among 200 patients included in the study, there were 127 cases of hypertension, 90 of dyslipidemia,
Table 3. Distribution of adherence levels according to the MAQ in the study population

MAQ levels Count Percent

\begin{tabular}{lll}
\hline MAQ hypertension $(\mathrm{n}=127)$ & & \\
Low level & 31 & 24.41 \\
Medium level & 27 & 21.26 \\
High level & 69 & 54.33 \\
MAQ hyperlipidemia $(\mathrm{n}=90)$ & & \\
Low level & 34 & 37.78 \\
Medium level & 25 & 27.78 \\
High level & 31 & 34.44 \\
MAQ diabetes mellitus $(\mathrm{n}=34)$ & & \\
Low level & 18 & 52.94 \\
Medium level & 3 & 8.82 \\
High level & 13 & 38.24 \\
\hline
\end{tabular}

and 41 of diabetes. The clinical and anthropometric data of the study population are shown in Tables 1 and 2 .

Antihypertensive drugs were prescribed to $65.46 \%$ of patients. The most common medications were angiotensin-converting enzyme inhibitors (ACEl; 45.50\%), followed by beta-adrenolytics (20\%), calcium channel blockers (7\%), angiotensin receptor blockers (ARBs; $7 \%$ ), and diuretics (4.5\%). According to the MAQ, $54.33 \%$ of the patients on antihypertensive drugs reported high adherence, $21.26 \%$ medium adherence, and $24.41 \%$ low adherence to the treatment. Lipid-lowering drugs were prescribed to $46.39 \%$ of the patients, all of whom were treated with statins. Among this group, $34.44 \%$ reported high adherence, $27.78 \%$ medium adherence, and $37.78 \%$ low adherence to pharmacotherapy. Antihyperglycemic drugs were prescribed to $17.53 \%$ of the patients. Based on the MAQ, 38.24\% of them reported high adherence, $8.82 \%$ medium adherence, and $52.94 \%$ low adherence. Due to the low number of patients on glucose-lowering drugs, the results were not analyzed statistically. The distribution of adherence levels according to the MAQ is presented in Table 3.

According to the ACDS, the majority of patients $(45.55 \%)$ received a score indicating medium adherence, $39.27 \%$ high adherence, and $16.75 \%$ low adherence to the treatment (Tab. 4).

Among the hypertensive patients who achieved blood pressure of $\leq 140 / 90 \mathrm{mmHg}$, only half declared high adherence to treatment while in the patients with elevated blood pressure ( $>140 / 90 \mathrm{mmHg}$ ) as many as $68.97 \%$ declared taking medication $100 \%$ of the time as prescribed. The high level of adherence was declared by $61.54 \%$ of the patients who reached the 
therapeutic goal of lipid-lowering therapy defined as LDL-C of $<2.6 \mathrm{mmol} / \mathrm{L}(<100 \mathrm{mg} / \mathrm{dL})$. On the other hand, among the patients whose LDL-C remained elevated, $23.44 \%$ declared high adherence to treatment. In patients with diabetes, who achieved fasting blood glucose concentration of $<100 \mathrm{mg} / \mathrm{dL}$, only $8.33 \%$ declared high adherence to medication while $83.33 \%$ declared low adherence. Due to the low number of patients on glucose-lowering drugs, the results were not analyzed statistically. The distribution of adherence levels according to the MAQ in relation to the treatment goal achievement is presented in Table 5.

In pharmacologically treated patients with hypertension and hyperlipidemia, as well as in the entire study population, the results of the MAQ were consistent with those of the ACDS (Tab. 6). The patients who declared high adherence in the MAQ obtained the highest mean ACDS scores, while the patients with low adherence, according to the MAQ, were characterized by the lowest mean ACDS scores.

\section{Discussion}

Poor adherence to recommended medication is a well-documented problem in the pharmacological treatment of chronic conditions, such as coronary artery

Table 4. Distribution of adherence levels according to the ACDS in the study population

\begin{tabular}{lcc}
\hline ACDS & $\mathbf{N}=\mathbf{1 9 4}$ & Percent \\
\hline Low (score < 21) & 32 & 16.50 \\
Medium (score 21-26) & 87 & 44.85 \\
High (score $>$ 26) & 75 & 38.65 \\
\hline
\end{tabular}

disease, diabetes, hyperlipidemia, hypertension [2-8, $15,16]$. It is hard to define medication adherence with a clear quantitative threshold below which a patient is classified as effectively non-adherent [17]. In the literature, the level of actual adherence $\geq 80 \%$ is typically considered relevant for the effectiveness of long-term pharmacotherapy [3,17-19]. In this study, the patients with indications for chronic pharmacotherapy due to hypertension, diabetes, and/or hyperlipidemia, were classified as having low, medium, or high declared adherence. It is extremely important to differentiate between the actual adherence and the declared adherence, as the latter is usually higher than the former [16, 34-37]. Insufficient or low adherence was defined as taking drugs $75 \%$ of the time or less according to the MAQ or as a score of $<21$ points in the ACDS [13-15]. In our study, $24.41 \%$ of the hypertensive patients and $37.78 \%$ of the patients with hyperlipidemia declared a low level of adherence in self-reported MAQ. Among the patients that were treated for hypertension and achieved the intended therapeutic goal of blood pressure $\leq 140 / 90 \mathrm{mmHg}$, only $50 \%$ declared high adherence in the MAQ. A slightly higher percentage was observed in the patients with hyperlipidemia; in this group, $61.54 \%$ of patients who reached the LDL-C of $<2.6 \mathrm{mmol} / \mathrm{L}$ ( $<100 \mathrm{mg} / \mathrm{dL}$ ) declared high adherence to the lipid-lowering treatment. The patients with high adherence reached their therapeutic goals significantly more often than those who were considered non-adherent to statin therapy $(p=0.0005)$.

Adherence to medications is a long process consisting of initiation, implementation, and persistence. The problem of non-initiation is estimated at $4-5 \%$ in clinical trials $[19,20]$. In routine clinical practice, over $20 \%$ of patients with hypertension, as well as those with dyslipidemia, never start their treatment [20-23]. However,

Table 5. Distribution of adherence levels according to the MAQ in relation to treatment goal achievement in the study population

\begin{tabular}{|c|c|c|c|c|}
\hline & \multicolumn{3}{|c|}{ MAQ } & \multirow[t]{2}{*}{ P-value } \\
\hline & Low & Medium & High & \\
\hline \multicolumn{5}{|l|}{ Hypertension $(n=127)$} \\
\hline $\mathrm{BP}>140 / 90 \mathrm{mmHg}$ & $17.24 \%$ & $13.79 \%$ & $68.97 \%$ & 0.1880 \\
\hline $\mathrm{BP} \leq 140 / 90 \mathrm{mmHg}$ & $26.53 \%$ & $23.47 \%$ & $50 \%$ & \\
\hline \multicolumn{5}{|l|}{ Hyperlipidemia $(n=90)$} \\
\hline $\mathrm{LDL}>2.6 \mathrm{mmol} / \mathrm{L}(>100 \mathrm{mg} / \mathrm{dL})$ & $48.44 \%$ & $28.13 \%$ & $23.44 \%$ & 0.0005 \\
\hline $\mathrm{LDL}<2.6 \mathrm{mmol} / \mathrm{L}(<100 \mathrm{mg} / \mathrm{dL})$ & $11.54 \%$ & $26.92 \%$ & $61.54 \%$ & \\
\hline \multicolumn{5}{|l|}{ Diabetes $(n=34)$} \\
\hline Glucose $>100 \mathrm{mg} / \mathrm{dL}$ & $36.36 \%$ & $9.09 \%$ & $54.55 \%$ & na* \\
\hline Glucose $<100 \mathrm{mg} / \mathrm{dL}$ & $83.33 \%$ & $8.33 \%$ & $8.33 \%$ & \\
\hline
\end{tabular}

*Due to the small number of patients on glucose-lowering drugs, the results were not analyzed statistically 
Table 6. Comparison of adherence levels with the score in the ACDS

\begin{tabular}{|c|c|c|c|c|}
\hline & \multicolumn{3}{|c|}{ ACDS } & \multirow[t]{2}{*}{ P-value } \\
\hline & $\mathbf{N}$ & Mean & SD & \\
\hline \multicolumn{5}{|c|}{ Hypertension ( $n=127$ ) } \\
\hline MAQ Low level & 31 & 21.29 & 3.95 & $<0.0001$ \\
\hline MAQ Medium level & 27 & 23.93 & 3.01 & \\
\hline MAQ High level & 69 & 26.01 & 2.34 & \\
\hline \multicolumn{5}{|c|}{ Hyperlipidemia ( $n=90$ ) } \\
\hline MAQ Low level & 34 & 22.74 & 4.63 & 0.0024 \\
\hline MAQ Medium level & 25 & 24.76 & 3.05 & \\
\hline MAQ High level & 31 & 26.19 & 2.50 & \\
\hline \multicolumn{5}{|l|}{ Diabetes $(n=34)$} \\
\hline MAQ Low level & 18 & 24.80 & 2.93 & na* \\
\hline MAQ Medium level & 3 & 20.00 & 1.73 & \\
\hline MAQ High level & 13 & 26.38 & 3.55 & \\
\hline \multicolumn{5}{|l|}{ Overall $(n=191)$} \\
\hline MAQ Low level & 64 & 22.25 & 4.09 & $<0.0001$ \\
\hline MAQ Medium level & 47 & 24.62 & 3.01 & \\
\hline MAQ High level & 80 & 25.97 & 2.61 & \\
\hline
\end{tabular}

*Due to the small number of patients on glucose-lowering drugs, the results were not analyzed statistically

it is the discontinuation of treatment that seems to be a greater issue [13, 22-26].

In many European countries, lipid control remains poor and most patients with dyslipidemia do not achieve the treatment goals recommended by the ESC guidelines [27]. This could be attributed to a high percentage of discontinuation of lipid-lowering treatment. Among patients with coronary heart disease in the EUROASPIRE IV survey, 90.4\% were on statins, and only $19.3 \%$ achieved values of LDL-C $<1.8 \mathrm{mmol} / \mathrm{L}$; statin was discontinued in $11.6 \%$ of cases [28]. Statins were also the most common medication class to be discontinued one year after myocardial infarction [24]. Only 50\% of patients after myocardial infarction continued the therapy with statins in one-year follow-up [27-29].

Adherence to pharmacotherapy of hypertension is necessary to achieve optimal levels of blood pressure control. Discontinuation of antihypertensive therapy is a common problem as more than $50 \%$ of patients stop their treatment after one year $[16,20]$. In addition, the rate of omitted doses is reported to be $10 \%$ per day [20]. A much higher adherence rate was observed in clinical trials with frequent clinical visits and where pill count was used to assess adherence [19]. In our study, there were no significant differences in achieving the therapeutic goal of blood pressure (BP $\leq 140 / 90 \mathrm{mmHg}$ ) in the groups with high (100\%), medium (90\%), and low adherence ( $\leq 75 \%)$ (26.53\% vs. $23.47 \%$ vs. $50.00 \%$; $\mathrm{p}=0.1880)$. Apart from low adherence, failure to achieve therapeutic goals might be related to inadequate therapy, as well as suboptimal dosage of prescribed medications.

As there are multiple reasons for non-adherence, efficient and simple tools are needed to determine patients' adherence in clinical practice [16, 26, 30-33]. Questionnaires are subjective methods and generally tend to overestimate true adherence when compared with more objective measures [34-37, 40]. The relatively low specificity and sensitivity may occur due to incorrect data reporting by patients. However, the simplicity, practicality, low cost, and real-time feedback have caused a widespread use of self-report questionnaires in clinical practice [16, 38, 40, 41]. Yet, adherence determined by questionnaires correlates with objective adherence measures and clinical outcomes, such as lipid levels, blood pressure, and blood glucose control [17]. Moreover, questionnaires can be useful tools to complement more objective methods of measurement as they may identify patients' concerns and reasons for non-adherence. Declarations regarding high and medium adherence in the MAQ and the ACDS were consistent; the patients with hyperlipidemia and hypertension with high adherence in the MAQ received an average of $>26$ points in the ACDS. The higher the adherence level declared in the MAQ, the higher was the score in the ACDS. The ACDS is designed to reflect various aspects of patient adherence [14, 15, 34-36]. 
This information can help implement an appropriate therapeutic plan, including additional personalized education and motivation methods [26, 39, 40].

\section{Limitations of the study}

The presented study had some limitations. The patients included in the study were not representative of all high cardiovascular-risk patients. Due to the small number of diabetic patients on glucose-lowering drugs, the results were not analyzed statistically. For evaluating medication adherence, only subjective methods in the form of self-report questionnaires were used. Patients' self-reported drug intake is often overestimated [34, 35]. Since there is no single method of choice in evaluating adherence, selecting at least two different methods (objective and subjective) can yield more reliable results. Finally, other factors that could have affected the therapeutic goals were not analyzed. In some cases, insufficient control of risk factors might have resulted from suboptimal doses of prescribed drugs.

\section{Conclusion}

Despite higher adherence to treatment in the patients with arterial hypertension compared to the patients with hyperlipidemia, the latter more often achieved the therapeutic goal. Declarations regarding high adherence to medication in the MAQ and in the ACDS are consistent in patients with hyperlipidemia and hypertension.

\section{Conflict of interest: None declared.}

\section{Funding: None declared.}

\section{References}

1. Bosworth HB, Granger BB, Mendys P, et al. Medication adherence: a call for action. Am Heart J. 2011; 162(3): 412-424, doi: 10.1016/j ahj.2011.06.007, indexed in Pubmed: 21884856.

2. Brown MT, Bussell JK. Medication adherence: WHO cares? Mayo Clin Proc. 2011 ; 86(4): 304-314, doi: 10.4065/mcp.2010.0575, indexed in Pubmed: 21389250

3. Pietrzykowski Ł, Michalski P, Kosobucka A, et al. Medication adherence and its determinants in patients after myocardial infarction. Sci Rep. 2020; 10(1): 12028, doi: 10.1038/s41598-020-68915-1, indexed in Pubmed: 32694522

4. Simpson SH, Eurich DT, Majumdar SR, et al. A meta-analysis of the association between adherence to drug therapy and mortality. BMJ. 2006; 333(7557): 15, doi: 10.1136/bmj.38875.675486.55, indexed in Pubmed: 16790458

5. Kubica J, Adamski P, Buszko K, et al. Rationale and design of the effectiveness of lower maintenanCe dose of TicagRelor early after myocardial infarction (ELECTRA) pilot study. Eur Heart J Cardiovasc Pharmacother. 2018; 4(3): 152-157, doi: 10.1093/ehjcvp/pvx032, indexed in Pubmed: 29040445.

6. DiMatteo MR. Variations in patients' adherence to medical recommendations: a quantitative review of 50 years of research. Med Care. 2004;
42(3): 200-209, doi: 10.1097/01.mlr.0000114908.90348.f9, indexed in Pubmed: 15076819

7. Tang L, Patao C, Chuang J, et al. Cardiovascular risk factor control and adherence to recommended lifestyle and medical therapies in persons with coronary heart disease (from the National Health and Nutrition Examination Survey 2007-2010). Am J Cardiol. 2013; 112(8): 1126-1132, doi: 10.1016/j.amjcard.2013.05.064, indexed in Pubmed: 23827404

8. Lam WY, Fresco P. Medication adherence measures: an overview. Biomed Res Int. 2015; 2015: 217047, doi: 10.1155/2015/217047, indexed in Pubmed: 26539470.

9. Visseren FLJ, Mach F, Smulders YM, et al. 2021 ESC Guidelines on cardiovascular disease prevention in clinical practice. Eur Heart J. 2021; 42(34): 3227-3337, doi: 10.1093/eurheartj/ehab484, indexed in Pubmed: 34458905

10. Chowdhury R, Khan H, Heydon E, et al. Adherence to cardiovascular therapy: a meta-analysis of prevalence and clinical consequences. Eur Heart J. 2013; 34(38): 2940-2948, doi: 10.1093/eurheartj/eht295, indexed in Pubmed: 23907142

11. De Bacquer D, Astin F, Kotseva $\mathrm{K}$, et al. EUROASPIRE IV and V surveys of the European Observational Research Programme of the European Society of Cardiology. Poor adherence to lifestyle recommendations in patients with coronary heart disease: results from the EUROASPIRE surveys. Eur J Prev Cardiol. 2021 [Epub ahead of print], doi: 10.1093/eurjpc/zwab115, indexed in Pubmed: 34293121.

12. Naderi SH, Bestwick JP, Wald DS. Adherence to drugs that prevent cardiovascular disease: meta-analysis on 376,162 patients. Am J Med. 2012; 125(9): 882-887.e1, doi: 10.1016/j.amjmed.2011.12.013, indexed in Pubmed: 22748400

13. Kubica A, Kasprzak M, Obońska K, et al. Discrepancies in assessment of adherence to antiplatelet treatment after myocardial infarction. Pharmacology. 2015; 95(1-2): 50-58, doi: 10.1159/000371392, indexed in Pubmed: 25592409

14. Buszko K, Obońska K, Michalski P, et al. The Adherence Scale in Chronic Diseases (ASCD). The power of knowledge: the key to successful patient - health care provider cooperation. Med Res J. 2016; 1(1): 37-42, doi: 10.5603/mri.2016.0006.

15. Kubica A, Kosobucka A, Michalski P, et al. The Adherence in Chronic Diseases Scale - a new tool to monitor implementation of a treatment plan. Folia Cardiol. 2017: 12: 19-26, doi: 10.5603/FC.2016.0105.

16. Hill M, Miller N, DeGeest S. Adherence and persistence with taking medication to control high blood pressure. J Am Soc Hypertens. 2011; 5(1): 56-63, doi: 10.1016/j.jash.2011.01.001.

17. Burnier M, Egan BM. Adherence in hypertension. Circ Res. 2019; 124(7): 1124-1140, doi: 10.1161/CIRCRESAHA.118.313220, indexed in Pubmed: 30920917.

18. Ho PM, Lambert-Kerzner A, Carey EP, et al. Multifaceted intervention to improve medication adherence and secondary prevention measures after acute coronary syndrome hospital discharge: a randomized clinical trial. JAMA Intern Med. 2014; 174(2): 186-193, doi: 10.1001/jamainternmed.2013.12944, indexed in Pubmed: 24247275.

19. Bansilal S, Castellano JM, Garrido E, et al. Assessing the impact of medication adherence on long-term cardiovascular outcomes. J Am Coll Cardiol. 2016; 68(8): 789-801, doi: 10.1016/j.jacc.2016.06.005, indexed in Pubmed: 27539170.

20. Vrijens B, Vincze G, Kristanto $P$, et al. Adherence to prescribed antihypertensive drug treatments: Iongitudinal study of electronically compiled dosing histories. BMJ. 2008; 336(7653): 1114-1117, doi: 10.1136/bmj.39553.670231.25, indexed in Pubmed: 18480115.

21. Kubica A, Kasprzak M, Obońska K, et al. Impact of health education on adherence to clopidogrel and clinical effectiveness of antiplatelet treatment in patients after myocardial infarction. Med Res J. 2015; 3(4): 154-159, doi: 10.5603/fmc.2015.0010.

22. Michalski P, Kasprzak M Siedlaczek M, et al. The impact of knowledge and effectiveness of educational intervention on readiness for hospital discharge and adherence to therapeutic recommendations in patients with acute coronary syndrome. Med Res J. 2020; 5(2): 72-78, doi: 10.5603/mrj.a2020.0023

23. Fischer MA, Choudhry NK, Brill G, et al. Trouble getting started: predictors of primary medication nonadherence. Am J Med. 2011; 124(11) 1081.e9-1081.e22, doi: 10.1016/j.amjmed.2011.05.028, indexed in Pubmed: 22017787

24. Pietrzykowski $Ł$, Kasprzak M, Michalski $P$, et al. Therapy discontinuation after myocardial infarction. J Clin Med. 2020; 9(12): 4109, doi 10.3390/jcm9124109, indexed in Pubmed: 33352811.

25. Kubica A, Pietrzykowski $Ł$. The therapeutic plan implementation in patients discharged from the hospital after myocardial infarction. Med Res J. 2021; 6(2): 79-82, doi: 10.5603/mrj.a2021.0024 
26. Kosobucka A, Pietrzykowski $Ł$, Michalski P, et al. Impact of readiness for discharge from the hospital on the implementation of the therapeutic plan. Med Res J. 2020; 5(4): 256-264, doi: 10.5603/mrj.a2020.0047.

27. Visseren FLJ, Mach F, Smulders YM, et al. ESC Scientific Document Group. 2021 ESC Guidelines on cardiovascular disease prevention in clinical practice. Eur Heart J. 2021; 42(34): 3227-3337, doi: 10.1093/eurheartj/ehab484, indexed in Pubmed: 34458905.

28. Reiner Ž, De Backer G. Fras Z, et al. EUROASPIRE Investigators. Lipid lowering drug therapy in patients with coronary heart disease from 24 European countries - findings from the EUROASPIRE IV survey Atherosclerosis. 2016; 246: 243-250, doi: 10.1016/j.atherosclerosis.2016.01.018, indexed in Pubmed: 26812002

29. Ho PM, Bryson CL, Rumsfeld JS. Medication adherence: its importance in cardiovascular outcomes. Circulation. 2009; 119(23): 3028-3035, doi: 10.1161/CIRCULATIONAHA.108.768986, indexed in Pubmed: 19528344

30. Kubica A, Adamski P, Baczkowska A, et al. The rationale for Multileve Educational and Motivational Intervention in Patients after Myocardia Infarction (MEDMOTION) project is to support multicentre randomized clinical trial Evaluating Safety and Efficacy of Two Ticagrelor-based De-escalation Antiplatelet Strategies in Acute Coronary Syndrome (ELECTRA - SIRIO 2). Med Res J. 2020; 5(4): 244-249, doi 10.5603/mrj.a2020.0043.

31. Kubica A Obońska K, Fabiszak T, et al Adherence to antiplatelet treatment with $\mathrm{P} 2 \mathrm{Y} 12$ receptor inhibitors. Is there anything we can do to improve it? A systematic review of randomized trials. Curr Med Res Opin. 2016; 32(8): 1441-1451, doi: 10.1080/03007995.2016.1182901, indexed in Pubmed: 27112628.

32. Kubica A, Obońska K, Kasprzak M, et al. Prediction of high risk o non-adherence to antiplatelet treatment. Kardiol Pol. 2016; 74(1) 61-67, doi: 10.5603/KPa2015.0117, indexed in Pubmed: 26101025

33. Kubica A, Kochman W, Bogdan M, et al. The influence of undergone percutaneous coronary interventions, and earlier hospitalizations with myocardial infarction on the level of knowledge and the effectiveness of health education in patients with myocardial infarction. Advances in Interventional Cardiology. 2009; 5(1): 25-30

34. Kubica A, Kasprzak M Siller-Matula J, et al. Time-related changes in determinants of antiplatelet effect of clopidogrel in patients after myocardial infarction. Eur J Pharmacol. 2014; 742: 47-54, doi: 10.1016/j. ejphar.2014.08.009, indexed in Pubmed: 25199965.

35. Kubica A Gruchała M, Jaguszewski M et al. Adherence to treatment - a pivotal issue in long-term treatment of patients with cardiovascular diseases. An expert standpoint. Med Res J. 2017; 2(4): 123-127, doi: 10.5603/mri.2017.0016.

36. Kubica A. Self-reported questionnaires for a comprehensive assessment of patients after acute coronary syndrome. Med Res J. 2019; 4(2): 106-109, doi: 10.5603/mrj.a2019.0021.

37. Kubica A, Kosobucka A, Michalski P, et al. Self-reported questionnaires for assessment adherence to treatment in patients with cardiovascular diseases. Med Res J. 2018: 2(4): 115-122, doi: 10.5603/mrj.2017.0015.

38. Kosobucka A, Michalski P. Pietrzykowski $€$, et al. Adherence to treatment assessed with the Adherence in Chronic Diseases Scale in patients after myocardial infarction. Patient Prefer Adherence. 2018; 12 : 333-340, doi: 10.2147/PPA.S150435, indexed in Pubmed: 29551891.

39. Pietrzykowski Ł, Kasprzak M, Michalski P, et al. The influence of patient expectations on adherence to treatment regimen after myocardial infarction. Patient Educ Couns. 2021 [Epub ahead of print], doi: 10.1016/j.pec.2021.05.030, indexed in Pubmed: 34059362

40. McDonald HP, Garg AX, Haynes RB. Interventions to enhance patient adherence to medication prescriptions: scientific review. JAMA 2002; 288(22): 2868-2879, doi: 10.1001/jama.288.22.2868, indexed in Pubmed: 12472329

41. Roumie CL, Greevy R, Wallston KA, et al. Patient centered primary care is associated with patient hypertension medication adherence. J Behav Med. 2011; 34(4): 244-253, doi: 10.1007/s10865-010-9304-6, indexed in Pubmed: 21161578. 